\title{
Possibility of lifetime measurement in a flowing medium with phase fluorometry
}

\author{
Jian-Bang Liu and Chang-Sheng Liu
}

\begin{abstract}
The possibility of lifetime measurement in a flowing medium with phase fluorometry is investigated theoretically. A 3-D time dependent partial differential equation of the number density of atoms (or molecules) in the upper level of the fluorescence transition is solved analytically, taking flow, diffusion, optical excitation, decay, Doppler shift, and thickness of the excitation light sheet into account. An analytical expression of the intensity of the fluorescence signal in the flowing medium is deduced. Conditions are given, in which the principle of lifetime measurement with phase fluorometry in the static sample cell can be used in a flowing medium. Key words: Lifetime, laser-induced fluorescence.
\end{abstract}

\section{Introduction}

Phase fluorometry has been successfully used in chemical, physical, and biological sciences for decades. It uses a temporally sinusoidally modulated continuous light source to excite the fluorescence of samples. The excited fluorescence intensity has been proved to be also temporally sinusoidally modulated but with a phase shift and a different modulation factor. The lifetime can be determined with the measured value of either the phase shift or the demodulation factor. Phase fluorometry has some advantages that make it promising: it avoids the necessity of generating short optical pulses; the intensity of the excitation light can be kept low to avoid nonlinear effects; the apparatus is easier to handle because one deals with the cw instead of the pulse, and the measurement can be precise and rapid. Several instruments are already commercially available.

In past decades, considerable literature on phase fluorometry has been published, ${ }^{1}$ and significant progress has been made. Multiple frequency modulation techniques and phase-resolved fluorescence spectroscopy (PRFS) have been adopted to resolve quantitatively individual spectra of a multiple component mixture. $^{2-10}$ Cross-correlation techniques have been successfully used to transpose phase and modulation information to the low frequency range where digital averaging techniques can be easily implemented.11,12

The authors are with Institute of Mechanics, Chinese Academy of Sciences, P.O. Box 2251, Beijing 100080, China.

Received 30 January 1990.

0003-6935/91/131688-07\$05.00/0.

(C) 1991 Optical Society of America.
Many advanced techniques have been combined with phase fluorometry to extend its application, e.g., synchrotron radiation, ${ }^{13,14}$ synchronous excitation, modelocked laser, cavity dumping, ${ }^{15-19}$ and optical fiber. ${ }^{20,21}$ A modulation frequency as high as $2 \mathrm{GHz}$ has been used, ${ }^{13-16}$ a mixture with as many as six components has been investigated, ${ }^{2}$ and a lifetime resolution in the picosecond time scale has been reached. ${ }^{22-24}$

Almost all the reported measurements with the phase fluorometer were done on a static sample until 1989 when Cabb and McGown demonstrated the first use of a multifrequency phase modulation spectrofluorometer for an on-line fluorescence lifetime determination in continuous flow conditions. ${ }^{25,26}$ For extending the application of phase fluorometry to a lifetime measurement in the flowing medium (e.g., gas and liquid flows), one must investigate the possible influences on the measurement results of phase fluorometry by the physical phenomena existing in fluids, e.g., flow, diffusion, and Doppler shift. Although the time scale of the lifetime measured by the phase fluorometer $\left(10^{-9} \mathrm{~s}\right)$ is often much less than the time scale of the flow, the measurement processes with phase fluorometers are essentially continuous. Therefore, possible influences by the physical phenomena mentioned above should be investigated before applying the phase fluorometer to fluids. Besides, the existing theory of phase fluorometry is based on the assumption of a uniform and steady sample. One must find in what condition the existing formulas connecting the lifetime to the directly measurable quantities (demodulation factor and phase shift) are applicable in fluids whose spatial distributions might be nonuniform and unsteady.

In this paper, a 3-D time dependent partial differential equation of the number density of the atoms (or 


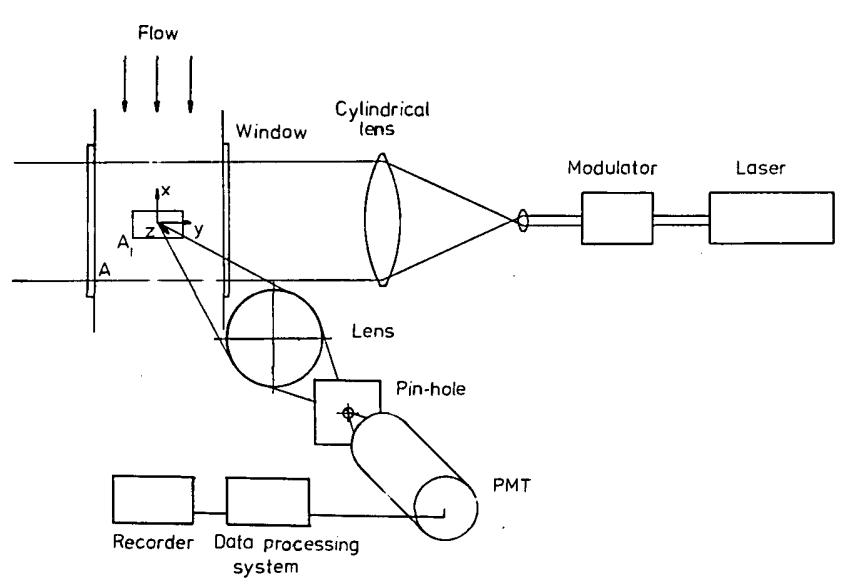

Fig. 1. Scheme of the experimental technique for lifetime measurement in the flowing medium with phase fluorometry.

molecules) in the upper level of the fluorescence transition is solved analytically, taking flow, diffusion, optical excitation, decay, Doppler shift, and thickness of the excitation laser sheet into account (Secs. II and III). The conditions in which the existing formulas derived for static samples are applicable to fluids are given (Sec. III). Finally, the influence of flow velocity, diffusion, Doppler shift, and thickness of the excitation laser sheet on the results of lifetime measurement is discussed (Sec. IV).

\section{Model and Equation}

Consider an experiment for lifetime measurement (Fig. 1). A parallel beam from a light source with an appropriate spectrum is focused into a thin sheet on the $x-y$ plane by a cylindrical lens irradiating the flowing medium. The intensity $I_{s}$ of the laser sheet is assumed to be uniform on the $x-y$ plane, Gaussian in the $z$-direction, and varying cosinusoidally with time $t$ :

$$
I_{s}=C_{a} \exp \left(-2 z^{2} / a_{\lambda}^{2}\right)[1-m \cos (2 \pi f t)] \quad 0 \leq m \leq 1,
$$

where $C_{a}$ is a constant and $a_{\lambda}$ is the laser sheet waist, i.e., a measure of the thickness of the laser sheet. The subscript $\lambda$ denotes that this parameter is related to the wavelength $\lambda$ of the excitation light, $m$ is the modulation factor of the excitation light, i.e., the ratio of the ac amplitude to the dc magnitude, and $f$ is the frequency. Under irradiation by the excitation laser sheet, the flowing medium fluoresces forming a luminescent fluid sheet. This luminescent sheet is imaged by a lens on a pinhole, through which the fluorescence signal is detected by a photomultiplier. After data processing, the lifetime of the flowing medium at the location determined by the pinhole and the excitation laser sheet is calculated with the formulas discussed below.

In Fig. $1 A$ is the whole field to be detected and is divided into a series of elementary areas $A_{i}(i=$ $1,2,3, \ldots)$. Within each $A_{i}$, the flow is assumed to be parallel, uniform, and steady; therefore, the temperature $T$, number density $n$, and three velocity components $v_{x}, v_{y}$, and $v_{z}$ are constant within each $A_{i}$ and each period of data acquisition, but in different $A_{i}$ and different times of data acquisition they might be different. The measured value of the lifetime within each $A_{i}$ and each period of data acquisition will correspond to certain $T, n$, and $v$ terms. If the flow does not satisfy this assumption, the measured value of the lifetime should correspond to the mean values of $T, n$, and $v$ within each $A_{i}$ and each period of data acquisition.

The fluorescence intensity is directly proportional to the number density $n_{u}(x, y, z, t)$ of the atoms (or molecules) in the upper level of the fluorescence transition. Within each $A_{i}$ and each period of data acquisition, $n_{u}(x, y, z, t)$ obeys following the 3-D time dependent partial differential equation

$$
\begin{aligned}
\frac{\partial n_{u}(x, y, z, t)}{\partial t}= & {\left[D\left(\frac{\partial^{2}}{\partial x^{2}}+\frac{\partial^{2}}{\partial y^{2}}+\frac{\partial^{2}}{\partial z^{2}}\right)\right.} \\
& \left.-v_{x} \frac{\partial}{\partial x}-v_{y} \frac{\partial}{\partial y}-v_{z} \frac{\partial}{\partial z}-\gamma\right] n_{u}(x, y, z, t) \\
& \quad+C_{b}\left(n, v_{y}\right) \exp \left(-2 z^{2} / a_{\lambda}^{2}\right)[1-m \cos (2 \pi f t)],
\end{aligned}
$$

where $D$ is the diffusion coefficient of the detected species in the flowing medium; $v_{x}, v_{y}$, and $v_{z}$ are the flow velocity components in the $x$-, $y$-, and $z$-directions, respectively; and $\gamma$ is the reciprocal lifetime:

$$
\gamma=1 / \tau \text {. }
$$

$C_{b}\left(n \cdot v_{y}\right)$ is a coefficient related to the excitation rate. For atoms with only two levels, $C_{b}$ is given by $C_{b}\left(n, v_{y}\right)$ $=n B C_{a}$. Here the number density of the atoms in the ground state is assumed to be approximately equal to the number density $n$ of atoms. Most of the experiments with phase fluorometers are carried out under weak excitation, and the number density $n_{u}$ of the upper level is negligible compared with that of the lower level. Therefore, this approximation is generally valid. $B$ is the absorption coefficient. In case of a nonzero flow velocity component in the propagation direction of the excitation light (the $y$-direction in Fig. 1), the absorption spectrum will be shifted with respect to the excitation light spectrum by the Doppler effect, and $B$ will be slightly changed. But in the directions perpendicular to the propagation direction of the excitation light (the $x$ - and $z$-directions in Fig. 1 ), the Doppler effect has no effect on $B$. Therefore, $B$ is dependent on $v_{y}$ but independent of $v_{x}$ and $v_{z}$. For molecules, $B$ is related to the particular upper and lower vibrational-rotational levels of the fluorescence transition and also a function of temperature. We will not discuss the detailed expression of $B$ further since $C_{b}$ will cancel later (Sec. IV).

The first through the third and the fourth through the sixth terms in the brackets in Eq. (2) describe the effects of diffusion and flow in the 3-D space, respectively; the seventh term describes the population decay of the upper level, which is assumed to be a single exponential. The last term on the right-hand side describes the effect of the optical excitation of the upper level.

Assume that the excitation laser sheet is bounded at $x=0$ but infinite in the $y$ - and $z$-directions and is 
switched on at time $t=0$. The initial and boundary conditions are

$$
\begin{aligned}
& n_{u}(x, y, z, 0)=n_{0} \exp \left(-2 z^{2} / a_{\lambda}^{2}\right) \quad n_{0}, \text { constant; } \\
& n_{u}(0, y, z, t)=n_{0} \exp \left(-2 z^{2} / a_{\lambda}^{2}\right) .
\end{aligned}
$$

In addition, the solution of $n_{u}(x, y, z, t)$ must be assumed to be finite to keep it unique.

\section{Solution}

To solve Eq. (2) with the initial and boundary conditions (4) and (5), a new variable is defined:

$$
\begin{aligned}
\Pi(x, y, z, t)=\exp \left(-\alpha_{x} x-\right. & \left.\alpha_{y} y-\alpha_{2} z-\beta t\right) \\
& \times\left[n_{u}(x, y, z, t)-n_{0} \exp \left(-2 z^{2} / a_{\lambda}^{2}\right)\right] .
\end{aligned}
$$

Using Eqs. (2), (4), (5), and (6), $\Pi(x, y, z, t)$ obeys Eq. (7) with initial and boundary conditions in Eqs. (8) and (9):

$$
\begin{aligned}
& \frac{\partial}{\partial t} \Pi(x, y, z, t)=D\left(\frac{\partial^{2}}{\partial x^{2}}+\frac{\partial^{2}}{\partial y^{2}}+\frac{\partial^{2}}{\partial z^{2}}\right) \Pi(x, y, z, t) \\
&+\exp \left(-\alpha_{x} x-\alpha_{y} y-\alpha_{z} z-\beta t-2 z^{2} / a_{\lambda}^{2}\right) \\
& \times {\left[-\gamma n_{0}+C_{b}\left(1-m \cos (2 \pi f t)-\frac{4 D n_{0}}{a_{\lambda}^{2}}\left(1-\frac{4 z^{2}}{a_{\lambda}^{2}}\right)+v_{z} \frac{4 n_{0} z}{a_{\lambda}^{2}}\right],\right.} \\
& \Pi(x, y, z, 0)=0=0, \\
& \Pi(0, y, z, t)=0
\end{aligned}
$$

where

$$
\begin{aligned}
& \alpha_{x}=\frac{v_{x}}{2 D}, \\
& \alpha_{y}=\frac{v_{y}}{2 D},
\end{aligned}
$$

After accomplishing the multiple integral in Eq. (14), the analytical expression of $n_{u}(x, y, z, t)$ can be obtained from Eq. (6). The 3-D distribution of the fluorescence intensity is given by

$$
I(x, y, z, t)=h \nu A n_{u}(x, y, z, t),
$$

where $h \nu$ is the photon energy emitted, and $A$ is the rate coefficient of spontaneous emission between the upper and lower levels. The position of the pinhole determines the spot position on the $x-y$ plane, the fluorescence emitted from which can reach the photomultiplier. In the $z$-direction, the fluorescence received by the photomultiplier is not from a certain point but from a line segment, which is the focus depth of the imaging optics. Therefore, the signal received by the photomultiplier should be integrated along the $z$-direction:

$$
S(x, y, t)=\int_{-\infty}^{\infty} \eta \frac{\Omega}{4 \pi} I(x, y, z, t) d z,
$$

where $\eta$ is the collection efficiency and $\Omega$ is the solid angle of the collection optics. In Eq. (16), the integral from $-\infty$ to $+\infty$ is used to replace the integral along the focus depth. The error introduced by this substitution is negligible, since the focus depth is generally much longer than the laser sheet waist, and the laser intensity outside the focus depth is very weak.

After a tedious mathematical derivation (see Appendix A), it can be shown that for

$$
t \gg \Delta t=\tau,
$$

the intensity of the fluorescence signal emitted by the flowing medium and received by the photomultiplier is given by

$$
\begin{gathered}
S(x, y, t)=\frac{C}{\gamma}-\left(\frac{C}{\gamma}-\sqrt{\frac{\pi}{2}} a_{\lambda} \eta \frac{\Omega}{4 \pi} h \nu A n_{0}\right) \exp \left[\left(\alpha_{x}-\sqrt{\alpha_{x}^{2}+\frac{\gamma}{D}}\right) x\right]-\frac{C m}{\sqrt{\gamma^{2}+(2 \pi f)^{2}}} \\
\times\left[1-2 \exp \left[\left(\alpha_{x}-A^{\prime}\right) x\right]\left[\cos \tan ^{-1}\left(\frac{2 \pi f}{\gamma}\right) \cos \left(B^{\prime} x-\epsilon\right)+\sin \tan ^{-1}\left(\frac{2 \pi f}{\gamma}\right) \sin \left(B^{\prime} x+\epsilon\right)\right]\right. \\
\left.+\exp \left[2\left(\alpha_{x}-A^{\prime}\right) x\right]\left(1+\frac{1}{2}\left\{\cos \left[2\left(B^{\prime} x-\epsilon\right)\right]-\cos \left[2\left(B^{\prime} x+\epsilon\right)\right]\right\}\right)\right]^{1 / 2} \\
\times \cos \left\{2 \pi f t-\tan ^{-1}\left[\frac{2 \pi f+\exp \left[\left(\alpha_{x}-A^{\prime}\right) x\right] \sqrt{(2 \pi f)^{2}+\gamma^{2}} \sin \left(B^{\prime} x+\epsilon\right)}{\gamma+\exp \left[\left(\alpha_{x}-A^{\prime}\right) x\right] \sqrt{(2 \pi f)^{2}+\gamma^{2}} \cos \left(B^{\prime} x-\epsilon\right)}\right]\right\}, \quad \text { (18) }
\end{gathered}
$$

$$
\begin{aligned}
& \alpha_{z}=\frac{v_{z}}{2 D}, \\
& \beta=-\frac{1}{4 D}\left(v_{x}^{2}+v_{y}^{2}+v_{z}^{2}\right)-\gamma .
\end{aligned}
$$

The solution of Eqs. (7), (8), and (9) is given by

$$
\begin{aligned}
\Pi(x, y, z, t) & =\int_{0}^{t}\left[\frac{1}{2 \sqrt{\pi D(t-\tau)}}\right]^{3} \int_{0}^{\infty} \int_{-\infty}^{+\infty} \int_{-\infty}^{+\infty}\left\{\exp \left[-\frac{(x-\xi)^{2}}{4 D(t-\tau)}\right]\right. \\
- & \left.\exp \left[-\frac{(x+\xi)^{2}}{4 D(t-\tau)}\right]\right\} \exp \left[-\frac{(y-\kappa)^{2}+(z-\zeta)^{2}}{4 D(t-\tau)}\right] \\
\times & \left\{-\gamma n_{0}+C_{b}[1-m \cos (2 \pi f \tau)]-\frac{4 D n_{0}}{a_{\lambda}^{2}}\left(1-\frac{4 \zeta^{2}}{a_{\lambda}^{2}}\right)+v_{z} \frac{4 n_{0} \zeta}{a_{\lambda}^{2}}\right\} \\
& \times \exp \left(-\alpha_{x} \xi-\alpha_{y} k-\alpha_{z} \zeta-\beta \tau-2 \zeta^{2} / a_{\lambda}^{2}\right) d \xi d \kappa d \zeta d \tau .
\end{aligned}
$$

where

$$
C=\sqrt{\frac{\pi}{2}} a_{\lambda} \eta \frac{\Omega}{4 \pi} h \nu A C_{b}\left(n, v_{y}\right)
$$

$$
\begin{aligned}
& A^{\prime}=\left[\frac{\sqrt{\left(\frac{v_{x}^{2}}{4 D^{2}}+\frac{\gamma}{D}\right)^{2}+\left(\frac{2 \pi f}{D}\right)^{2}}+\frac{v_{x}^{2}}{4 D^{2}}+\frac{\gamma}{D}}{2}\right]^{1 / 2}, \\
& B^{\prime}=\left[\frac{\sqrt{\left(\frac{v_{x}^{2}}{4 D^{2}}+\frac{\gamma}{D}\right)^{2}+\left(\frac{2 \pi f}{D}\right)^{2}}-\frac{v_{x}^{2}}{4 D^{2}}-\frac{\gamma}{D}}{2}\right]^{1 / 2},
\end{aligned}
$$




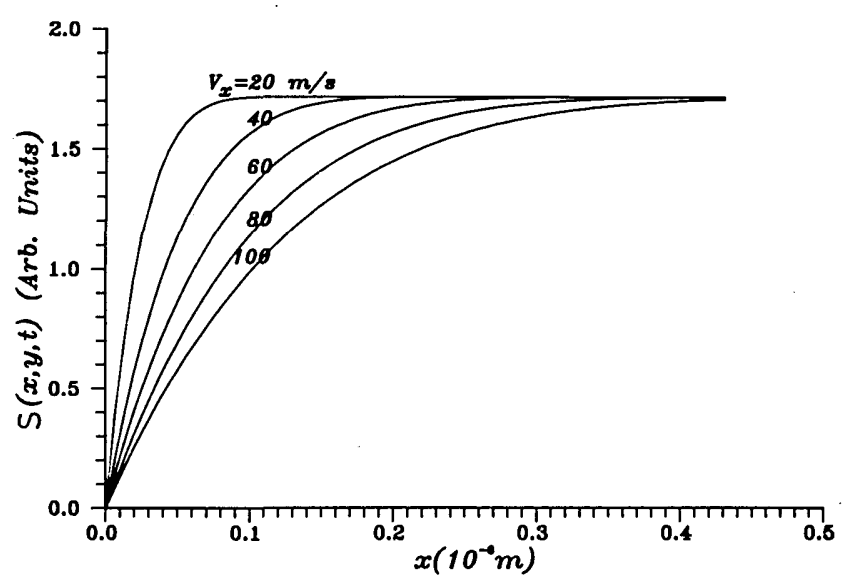

Fig. 2. Dependences of the intensity of the fluorescence signal $S(x, y, t)$ on $x$ with different $v_{x}: f=10^{8} \mathrm{~Hz} ; \tau=10^{-9} \mathrm{~s} ; D=10^{-9} \mathrm{~m}^{2} / \mathrm{s} ; t$ $=5 \times 10^{-9} \mathrm{~s} ; n_{0}=0 ; m=1$.

$$
\epsilon=\tan ^{-1}\left[\frac{\frac{B^{\prime}}{\left(\alpha_{x}+A^{\prime}\right)^{2}+B^{\prime 2}}-\frac{B^{\prime}}{\alpha_{x}-A^{\prime}}+\frac{\alpha_{x}+A^{\prime}}{\left(\alpha_{x}-A^{\prime}\right)^{2}+B^{\prime 2}}+\frac{B^{2}}{\left(\alpha_{x}+A^{\prime}\right)^{2}+B^{\prime 2}}}{\left(\alpha_{x}\right.}\right] .
$$

Physically, the inequality (17) means that after a relaxation time $\Delta t$, whose value is equal to the lifetime $\tau$ of the fluorescence transition, the fluorescence signal $S(x, y, t)$ obeys Eq. (18). In fact $\Delta t=\tau$ implies that Eq. (18) describes only the signal received from the flowing medium, which is thoroughly relaxed. The dependences of $S(x, y, t)$ on $x$ with different $v_{x}$ are shown in Fig. 2, from which the effects of boundary and velocity on the fluorescence signal $S(x, y, t)$ can be seen. Note that

$$
A^{\prime} \geq \sqrt{\frac{v_{x}^{2}}{4 D^{2}}+\frac{\gamma}{D}} .
$$

In the case of

$$
x \gg \Delta x=\left(\sqrt{\frac{v_{x}^{2}}{4 D^{2}}+\frac{\gamma}{D}}-\frac{v_{x}}{2 D}\right)^{-1},
$$

$S(x, y, t)$ reduces to

$$
S(x, y, t)=\frac{C}{\gamma}-\frac{C m}{\sqrt{\gamma^{2}+(2 \pi f)^{2}}} \cos \left[2 \pi f t-\tan ^{-1}\left(\frac{2 \pi f}{\gamma}\right)\right] .
$$

According to Eq. (24), the phase shift of $S(x, y, t)$ with respect to the excitation process is given by

$$
\Phi=\tan ^{-1}\left(\frac{2 \pi f}{\gamma}\right)
$$

and the demodulation factor $M$, which is the ratio of the modulation factor of $S(x, y, t)$ to that of the laser sheet intensity $I_{s}$, is given by

$$
M=\frac{\gamma}{\sqrt{\gamma^{2}+(2 \pi f)^{2}}} .
$$

Both $\Phi$ and $M$ are directly measurable. The lifetime $\tau$ $=1 / \gamma$ can be determined from either Eq. (25) or (26):

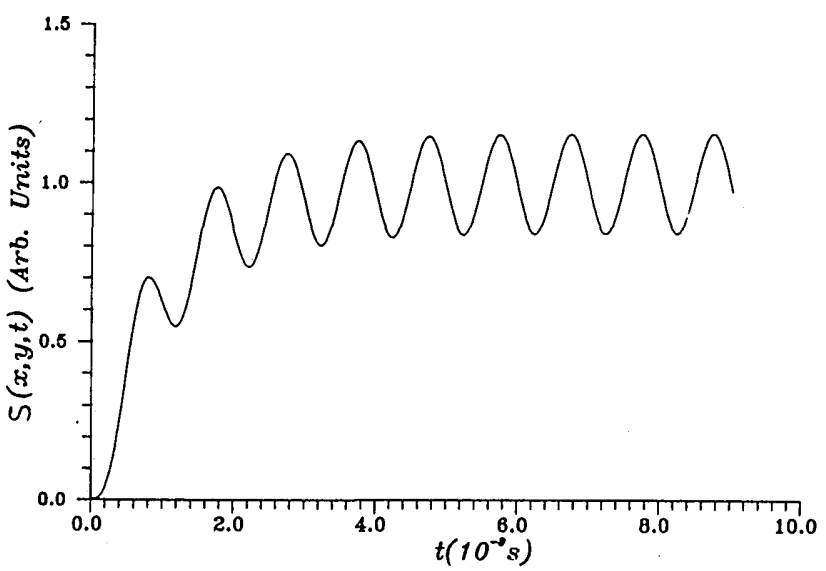

Fig. 3. Dependence of the intensity of the fluorescence signal on $t$ : $f=10^{9} \mathrm{~Hz} ; \tau=10^{-9} \mathrm{~s} ; n_{0}=0 ; m=1$.

$$
\begin{aligned}
& \tau=\frac{1}{2 \pi f} \tan ; \\
& \tau=\frac{1}{2 \pi f} \sqrt{\frac{1}{M^{2}}-1} .
\end{aligned}
$$

Equations (27) and (28) are the same as those commonly used in phase fluorometry, ${ }^{27}$ but this time they are shown to be valid for a flowing medium. Note that $S(x, y, t)$ in the flowing medium is inherently very complicated as shown in Eq. (18). The lifetime $\tau$ can be expressed with the same simple formulas as used in the static medium only in conditions when $t \gg \Delta t$ and $x \gg$ $\Delta x$.

In Eqs. (24) and (18), the mean values (the dc component), amplitude of modulation, and phase shift of $S(x, y, t)$ are independent of time $t$, because an approximation $t \gg \Delta t$ was adopted. In the period immediately after switching on the light source $(t<\Delta t)$, some or all of these quantities should vary with time. To clarify this point, Eq. (2) is solved again but for an infinite 3-D space (see Appendix B). With such an assumption, the equation can be solved analytically without using the approximation $t \gg \Delta t$. The result [Eq. (41)] shows that the mean value of $S(x, y, t)$ does depend on time. The effect of the initiation of the light source on the fluorescence signal $S(x, y, t)$ is shown in Fig. 3. For the case of $x \gg \Delta x$ and $t \gg \Delta t$, Eqs. (24) and (41) give the same solution.

\section{Discussion and Conclusion}

(A) Equations (27) and (28), which were derived for the flowing medium, are independent of $v_{x}, v_{y}, v_{z}, D, a_{\lambda}$, and $C\left(n, v_{y}\right)$, which implies that the measured values of lifetime by phase fluorometry in flowing medium in conditions $i \gg \Delta t$ and $x \gg \Delta x$ are not influenced by flow velocity, diffusion, thickness of the laser sheet, and Doppler shift. This can be understood by considering the assumptions in Sec. II and the approximations adopted: (a) within each elemental area $A_{i}$ and each period of data acquisition, the flow is assumed to 
be parallel, uniform, and steady; (b) the intensity of the laser sheet is uniform on the $x-y$ plane; and (c) the approximations $x \gg \Delta x$ and $t \gg \Delta t$, by which the effects of the boundary and the initiation of the laser sheet on the flow were made negligible. Consequently, although the flow continuously changes the part of the flowing medium observed by the photomultiplier, the fluorescence signal received by the photomultiplier remains unchanged; i.e., the flow velocity does not influence the fluorescence signal $S(x, y, t)$. For the same reason, there is no gradient of number density existing within an elemental area $A_{i}$ in the $x$ - and $y$ directions; i.e., there is no diffusion process taking place within an $A_{i}$ in the $x$ - and $y$-directions. Although there is a gradient of number density in the $z$ direction caused by the Gaussian distribution of the intensity of the laser sheet, owing to the integration along the $z$-direction [Eq. (16)], the diffusion in the $z$ direction still has no influence on the fluorescence signal received by the photomultiplier. That $v$ and $D$ do not influence the measured value of lifetime implies that the phase fluorometry could be used for a very wide range of velocity $v$ and diffusion coefficient $D$. The latter is inversely proportional to $n$, if the flowing medium is a simple substance, or to the total density of the flowing medium, if it is a mixture. ${ }^{28}$ Therefore, the phase fluorometer might be used in case of high velocity and low density. But it should be remembered that $\Delta x$, which is one of the conditions defining the applicable range of the phase fluorometer in the flowing medium, is still dependent on $v$ and $D$ [Eq. (23)]. $C\left(n, v_{y}\right)$ is canceled during the derivation of the modulation factor $M$ [Eq. (26)]. Therefore, the laser sheet waist $a_{\lambda}$ and the Doppler effect have no influence on the measurement results.

(B) According to Eq. (23) $\Delta x$ describes how far apart two adjacent elementary areas $A_{i}$ and $A_{j}$ should be, where $A_{i}$ and $A_{j}$ are the elementary areas in which $n(x, y, z, t)=n_{0} \exp \left(-2 z^{2} / a_{\lambda}^{2}\right)$ and $n(x, y, z, t)$ obey Eq. (24), respectively. Similarly, according to Eq. (17), $\Delta t$ describes how long it should take for an elementary area starting from a state in which $n(x, y, z, t)=n_{0}$ $\exp \left(-2 z^{2} / a_{\lambda}^{2}\right)$ to reach another state in which $n(x, y, z, t)$ obeys Eq. (24). In other words, the measurement principle of phase fluorometry is valid in a flowing medium, provided the dimensions of the elementary areas, in which the flow can be considered parallel and uniform, are much greater than $\Delta x$ and the periods, in which the state of the flowing medium can be considered steady, are much longer than $\Delta t . \Delta x$ depends on the physical characteristics ( $\tau$ and $D$ ) and the velocity of the flowing medium. $\Delta t$ depends on the lifetime $\tau$ of the flowing medium only. Dependences of $\Delta x$ and $\Delta t$ on $\tau$ with different $v_{x}$ are shown in Fig. 4. Dependences of $\Delta x$ on $\tau$ with different $D$ are shown in Fig. 5. Generally $\Delta x$ and $\Delta t$ have the same orders of magnitude as $v_{x} \tau$ and $\tau$, respectively, which are satisfied for many practical applications.

(C) In principle, measurement of the 2-D distribution of the lifetime is possible if a 2-D detector with the necessary temporal resolution is available. In this

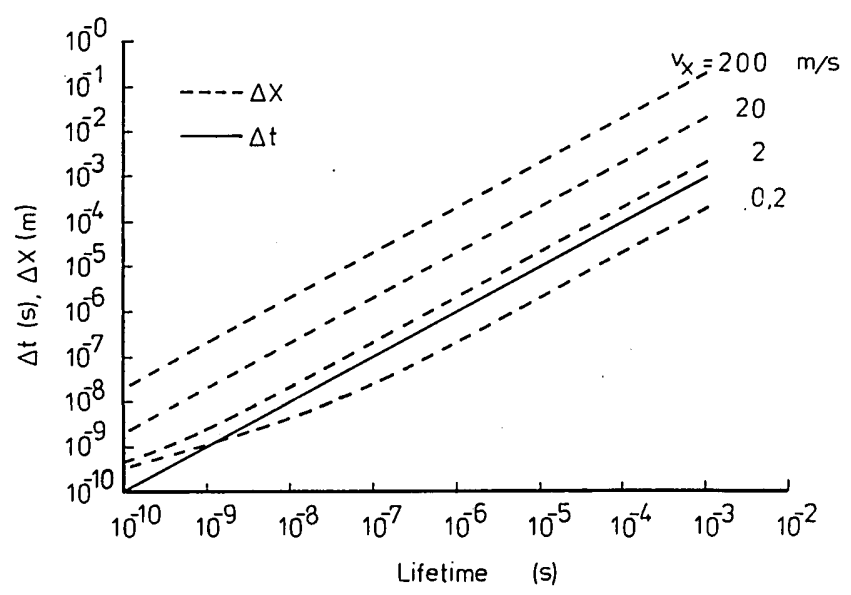

Fig. 4. Dependences of $\Delta x$ and $\Delta t$ on lifetime $\tau$ with different $v_{x}$ terms: $D=10^{-9} \mathrm{~m}^{2} / \mathrm{s}$.

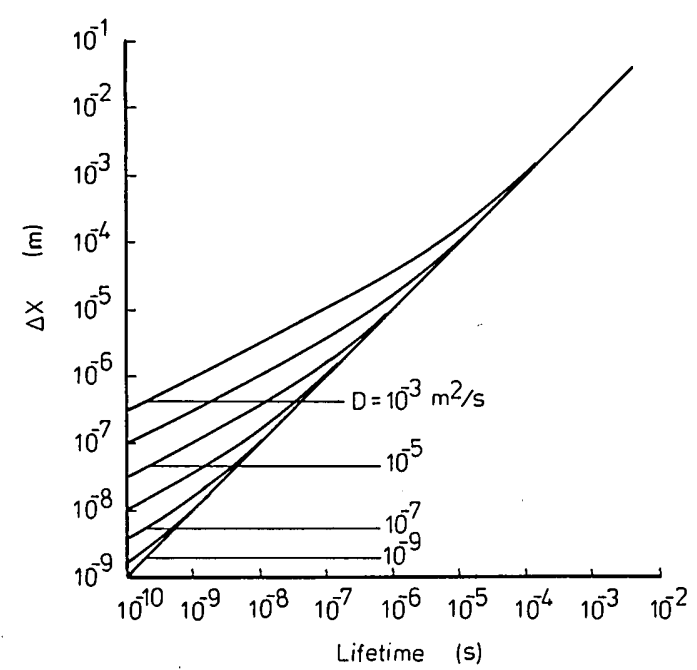

Fig. 5. Dependences of $\Delta x$ on lifetime $\tau$ with different $D: v_{x}=10$ $\mathrm{m} / \mathrm{s}$.

case, the 2-D detector should be used instead of the photomultiplier and the pinhole. The output electrical signal is acquired, processed, and recorded by a data processing and computer system.

In summary, the lifetime measurement in the flowing medium by phase fluorometry should be possible, provided the conditions $x \gg \Delta x$ and $t \gg \Delta t$ are fulfilled. The flow velocity, diffusion, Doppler shift, and thickness of the excitation light sheet do not influence the measured values of the lifetime.

The authors thank Zheng Zhe-Min, Wu ChengKang, Xie Bo-Min, and Chow Quantie for fruitful discussions. The authors thank the National Natural Science Foundation of China and the Third World Academy of Sciences for their support. 
Using Eqs. (6), (14), and (16), $S(x, y, t)$ is given by

To prove Eq. (31), after accomplishing the integral of $\tau, I^{\prime}$ becomes

$$
\begin{aligned}
S(x, y, t)=\eta \frac{\Omega}{4 \pi} h \nu A & \int_{-\infty}^{+\infty}\left(\exp \left(\alpha_{x} x+\alpha_{y} y+\alpha_{z} z+\beta t\right) \int_{0}^{t} \int_{0}^{\infty} \int_{-\infty}^{+\infty} \int_{-\infty}^{+\infty}\left[\frac{1}{2 \sqrt{\pi D(t-\tau)}}\right]^{3}\right. \\
& \times\left\{\exp \left[-\frac{(x-\xi)^{2}}{4 D(t-\tau)}\right]-\exp \left[-\frac{(x+\xi)^{2}}{4 D(t-\tau)}\right]\right\} \exp \left\{-\frac{(y-\kappa)^{2}+(z-\zeta)^{2}}{4 D(t-\tau)}-\alpha_{x} \xi-\alpha_{y} k-\alpha_{z} \zeta-\beta \tau-2 \zeta^{2} / a_{\lambda}^{2}\right\} \\
& \left.\times\left\{-\gamma n_{0}+C[1-m \cos (2 \pi f \tau)]-\frac{4 D n_{0}}{a_{\lambda}^{2}}\left(1-\frac{4 \zeta^{2}}{a_{\lambda}^{2}}\right)+v_{z} \frac{4 n_{0}}{a_{\lambda}^{2}} \zeta\right\} d \xi d x d \zeta d \tau+n_{0} \exp \left(-2 z^{2} / a_{\lambda}^{2}\right)\right) d z .
\end{aligned}
$$

For accomplishing the multiple integral, the following two equations [(30) and (31)] must be proved in advance:

$$
\begin{gathered}
\int_{0}^{\infty} \exp \left(-\alpha_{x} \xi\right)\left\{\exp \left[-\frac{(x-\xi)^{2}}{4 D(t-\tau)}\right]-\exp \left[-\frac{(x+\xi)^{2}}{4 D(t-\tau)}\right]\right\} d \xi \\
=\frac{4 \sqrt{D(t-\tau)}}{\sqrt{\pi}} \int_{0}^{\infty} \frac{\exp \left[-D(t-\tau) \sigma^{2}\right] \sigma \sin \sigma x}{\sigma^{2}+\alpha_{x}^{2}} d \sigma, \quad(30) \\
I^{\prime} \equiv \exp (\beta t) \int_{0}^{\infty} \frac{\sigma \sin \sigma x}{\sigma^{2}+\alpha_{x}^{2}} \int_{0}^{t} \exp \left[D(t-\tau)\left(-\sigma^{2}+\alpha_{y}^{2}+\alpha_{z}^{2}\right)-\beta \tau\right] d \tau d \sigma \\
=\frac{\pi}{2 \gamma}\left[\exp \left(-\alpha_{x} x\right)-\exp \left(-\sqrt{\alpha_{x}^{2}+\frac{\gamma}{D}} x\right)\right],
\end{gathered}
$$

provided $t \gg \Delta t$.

A. Derivation of Eq. (30)

To prove Eq. (30), use the convolution theorem of the Fourier transform ${ }^{29}$ :

$$
\int_{-\infty}^{+\infty} \dot{\bar{f}}(\sigma) \bar{g}(\sigma) \exp (-i \sigma x) d \sigma=\int_{-\infty}^{+\infty} g(s) f(x-s) d s,
$$

where $\vec{f}(\sigma)$ and $\vec{g}(\sigma)$ are the Fourier transforms of the functions $f(\xi)$ and $g(\xi)$, respectively, e.g.,

$$
\bar{f}(\sigma)=\frac{1}{\sqrt{2 \pi}} \int_{-\infty}^{+\infty} f(\xi) \exp (-i \sigma \xi) d \xi \text {. }
$$

From Eqs. (32) and (33), it can be obtained that

$$
\int_{0}^{+\infty} f_{s}(\sigma) g_{c}(\sigma) \sin \sigma x d \sigma=\frac{1}{2} \int_{0}^{\infty} f(s)[g(|x-s|)-g(x+s)] d s,
$$

$$
\begin{aligned}
I^{\prime}=-\int_{0}^{\infty} \frac{\sigma \sin \sigma x}{\left(\sigma^{2}+\alpha_{x}^{2}\right)\left(D \sigma^{2}+D \alpha_{x}^{2}+\gamma\right)} \\
\quad \times\left\{1-\exp \left[-\left(D \sigma^{2}+D \alpha_{x}^{2}+\gamma\right) t\right]\right\} d \sigma
\end{aligned}
$$

Note that $D \sigma^{2}+D \alpha_{x}^{2}+\gamma \geq \gamma$ in the case of $t \gg \Delta t$, using inequality

$$
\exp \left[-\left(D \sigma^{2}+D \alpha_{x}^{2}+\gamma\right) t\right] \ll 1
$$

the factor $\exp \left(D \sigma^{2}+D \alpha_{x}^{2}+\gamma\right) t$ in Eq. (39) can be neglected and Eq. (31) can be proved easily.

With Eqs. (30) and (31), the multiple integral can be accomplished and the final expression of $S(x \cdot y, t)[\mathrm{Eq}$. (18)] can be obtained.

\section{Appendix B: Solution of Eq. (2) for Infinite Three- Dimensional Space}

To investigate the temporal behavior of $S(x, y, t)$ in the period immediately after switching on the light source, we solve Eq. (2) in an infinite 3-D space. The omission of the boundary condition (4) makes it possible to solve Eq. (2) analytically without using the approximation $t \gg \Delta t$. Physically this implies only the solution of $S(x, y, t)$ as far as the area far from the boundary is concerned. With this assumption, $\Pi(x, y, z, t)$ is given by

$$
\begin{array}{r}
\Pi(x, y, z, t)=\int_{0}^{t} \int_{-\infty}^{+\infty} \int_{-\infty}^{+\infty} \int_{-\infty}^{+\infty}\left(\frac{1}{2 \sqrt{\pi D(t-\tau)}}\right)^{3} \exp \left[-\frac{(x-\xi)^{2}+(y-\kappa)^{2}+(z-\zeta)^{2}}{4 D(t-\tau)}-\alpha_{x} \xi-\alpha_{y} \kappa-\alpha_{z} \zeta-\beta \tau-2 \zeta^{2} / a_{\lambda}^{2}\right] \\
\times\left\{-\gamma n_{0}+C_{b}[1-m \cos (2 \pi f \tau)]-\frac{4 D n_{0}}{a_{\lambda}^{2}}\left(1-\frac{4 \zeta^{2}}{a_{\lambda}^{2}}\right)+v_{z} \frac{4 n_{0}}{a_{\lambda}^{2}} \zeta\right\} d \xi d \kappa d \zeta d \tau
\end{array}
$$

where

$$
\begin{aligned}
& f_{s}(\sigma)=\sqrt{\frac{2}{\pi}} \int_{0}^{\infty} f(\xi) \sin \sigma \xi d \xi \\
& g_{c}(\sigma)=\sqrt{\frac{2}{\pi}} \int_{0}^{\infty} g(\xi) \cos \sigma \xi d \xi .
\end{aligned}
$$

Let the functions $f(\xi)$ and $g(\xi)$ in Eqs. (35) and (36) be

$$
\begin{aligned}
& f(\xi)=\exp \left(-\alpha_{x} \xi\right) \\
& g(\xi)=\exp \left[-\frac{\xi^{2}}{4 D(t-\tau)}\right]
\end{aligned}
$$

respectively; then Eq. (30) can be proved.
The integration of the multiple integral in Eq. (40) can be accomplished without using any additional approximation or assumption. The final result is given by

$$
\begin{array}{r}
S(x, y, t)=\frac{C}{\gamma}-\left[\frac{C}{\gamma}-\sqrt{\frac{\Pi}{2}} a_{\lambda} \eta \frac{\Omega}{4 \pi} h \nu A n_{0}-C m \frac{\gamma}{\gamma^{2}+(2 \pi f)^{2}}\right] \\
\times \exp (-\gamma t)-\frac{C m}{\sqrt{\gamma^{2}+(2 \pi f)^{2}}} \cos \left[2 \pi f t-\tan ^{-1}\left(\frac{2 \pi f}{\gamma}\right)\right]
\end{array}
$$

For the time $t \gg \Delta t$, Eq. (41) becomes the same as Eq. (24). 


\section{References}

1. D. M. Jameson, E. Gratton, and R. D. Hall, "The Measurement and Analysis of Heterogeneous Emission by Multifrequency Phase and Modulation Fluorometry," Appl. Spectrosc. Rev. 20, 55-106 (1984).

2. K. Nithipatikom and L. B. McGown, "Five and Six-Component Determination Using Phase-Resolved Fluorescence Spectroscopy and Synchronous Excitation," Appl. Spectrosc. 41, 395-399 (1987).

3. K. Nithipatikom and L. B. McGown, "Multidimensional Dataformat for Phase Resolved Fluorometry Multicomponent Determinations Using Synchronous Excitation and Emission Spectra," Anal. Chem. 58, 2469-2473 (1986).

4. S. M. Keating-Nakamoto, H. Cherek, and J. R. Lakowicz, "Resolution of Multicomponent Fluorescence Emission by Phase Sensitive Detection at Multiple Modulation Frequencies," Anal. Chem. 59, 271-278 (1987).

5. L. B. McGown, "Determination of Fluorescence Lifetime and Heterogeneity Analysis Using Time-Independent Phase-Resolved Intensity Measurements," Anal. Instrum. 14, 251-265 (1985).

6. F. V. Bright and L. B. McGown, "Phase-Resolved Fluomatric Determinations of Four-Component Systems Using Two Modulation Frequencies," Anal. Chem. 57, 2877-2880 (1985).

7. F. V. Bright and L. B. McGown, "Four-Component Determinations Using Phase-Resolved Fluorescence Spectroscopy," Anal. Chem. 57, 55-59 (1985).

8. L. B. McGown and F. V. Bright, "Comparison of Phase-Resolved and Steady-State Fluorometric Multicomponent Determinations Using Wavelength Selection," Anal. Chem. 56, 21952199 (1984).

9. L. B. McGown, "Phase-Resolved Fluorimetric Determination of Two Albumin-Bound Fluorescein Species," Anal. Chim. Acta 157, 327-332 (1984).

10. L. B. McGown and F. V. Bright, "Simultaneous Two-Component Determinations by Phase-Resolved Fluorescence Spectroscopy," Anal. Chim. Acta 169, 117-123 (1985).

11. R.D. Spencer and G. Weber, "Measurements of Subnanosecond Fluorescence Lifetimes with a Cross-Correlation Phase Fluorometer," Ann. NY Acad. Sci. 158, 361-376 (1969).

12. J. R. Lakowicz and H. Cherck, "Phase-Sensitive Fluorescence Spectroscopy: A New Method to Resolve Fluorescences Lifetimes or Emission Spectra of Components in a Mixture of Fluorophors," J. Biochem. Biophys. Methods 5, 19-35 (1981).

13. E. Gratton, D. M. Jameson, N. Rosato, and G. Weber, "Multifrequency Cross-Correlation Phase Fluorometer Using Synchrotron Radiation," Rev. Sci. Instrum. 55, 486-494 (1984).

14. P. Sebban and I. Moya, "Fluorescence Lifetime Spectra of in vivo Bacteriochlorophyll at Room Temperature," Biochim. Biophys. Acta 722, 436-442 (1983).
15. J. R. Lakowicz, G. Laczko, and I. Gryczynski, "2-GHz Frequency-Domain Fluorometer,” Rev. Sci. Instrum. 57, 2499-2506 (1986).

16. J. R. Lakowicz, M. L. Johnson, I. Gryczynski, N. Joshi, and G. Laozko, "Transient Effects in Fluorescence Quenching Measured by $2-\mathrm{GHz}$ Frequency Domain Fluorometry," J. Phys. Chem. 91, 3277-3285 (1987).

17. K. Nithipatikom and L. B. McGown, "Elimination of Scatter Background in Synchronous Excitation Spectrofluorometry by the Use of Phase-Resolved Fluorescence Spectroscopy," Anal. Chem. 58, 3145-3148 (1986).

18. M. J. Wirth and S.-H. Chon, "Phase-Resolved Subnanosecond Spectroscopy Using the Beat Frequency from Mode-Locked Laser," Appl. Spectrosc. 42, 483-486 (1988).

19. F. V. Bright, D. A. Daniel, A. Wilson, and G. M. Hieftje, "Determination of Subnanosecond Fluorescence Lifetimes with a UHF Television Tuner and a CW or Mode-Locked Laser," Appl. Spectrosc. 40, 683-687 (1986).

20. F. V. Bright, "A New Fiber-Optic-Based Multifrequency PhaseModulation Fluorometer," Appl. Spectrosc. 42, 1531-1537 (1988).

21. F. V. Bright, C. A. Monnig, and G. M. Hiegtje, "Rapid Frequency-Scanned Fiber-Optic Fluorometer Capable of Subnanosecond Lifetime Determination," Anal. Chem. 58, 3139-3144 (1986).

22. H.-P. Hear and M. Hauser, "Phase Fluorometer for Measurement of Picosecond Processes," Rev. Sci. Instrum. 49, 632-633 (1978).

23. E. R. Menzel and Z. D. Popovic, "Picosecond-Resolution Fluorescence Lifetime Measuring System with CW Laser and a Radio," Rev. Sci. Instrum. 49, 39-44 (1978).

24. J. R. Lakowicz, G. Laczko, I. Gryczynski, and H. Cherck, "A 2 $\mathrm{GHz}$ Frequency-Domain Fluorometer; Picosecond Resolution of Tyrosine Fluorescence and Anisotropy Decays," Proc. Soc. Photo-Opt. Instrum. Eng. 712, 110-116 (1987).

25. W. T. Cobb and L. B. McGown, "Multifrequency Phase-Modulation Fluorescence Lifetime Determination On-the-Fly in HPLC," Appl. Spectrosc. 43, 1363-1367 (1989).

26. W. T. Cobb and L. B. McGown, "On-Line Fluorescence Lifetime Detection for Chromatographic Peak Resolution," Anal. Chem. 62, 186-189 (1990).

27. J. Yquerabide and E. Yquerabide, "Nanosecond Fluorescence Spectroscopy," in Optical Techniques in Biological Research, D. L. Rousseau, Ed. (Academic, Orlando, FL, 1984), pp. 181187.

28. R. D. Present, Kinetic Theory of Gases (McGraw-Hill, New York, 1958).

29. J. W. Goodman, Introduction to Fourier Optics (McGraw-Hill, New York, 1968). 